\title{
Analysis of microvascular density in early gastric carcinoma using magnifying endoscopy with narrow-band imaging
}

Authors

Institutions
Masashi Kawamura' ${ }^{1}$, Hiroshi Naganuma², Rie Shibuya² ${ }^{2}$ Tatsuya Kikuchi ${ }^{1}$, Yoshitaka Sakai ${ }^{1}$, Futoshi Nagasaki ${ }^{1}$, Eiki Nomura ${ }^{1}$, Noriaki Suzuki ${ }^{1}$, Eri Saito ${ }^{1}$

${ }^{1}$ Department of Gastroenterology, Sendai City Hospital, Miyagi, Japan

2 Department of Pathology, Sendai City Hospital, Miyagi, Japan submitted

23. December 2015

accepted after revision

17. May 2016

\section{Bibliography}

DOI http://dx.doi.org/

10.1055/s-0042-110095

Published online: 9.8.2016

Endoscopy International Open

2016; 04: E832-E837

(C) Georg Thieme Verlag KG

Stuttgart · New York

E-ISSN 2196-9736

\section{Corresponding author}

\section{Kawamura, MD, PhD}

Department of

Gastroenterology

Sendai City Hospital

1-1-1 Asutonagamachi

Taihaku-ku

Sendai

Miyagi 982-8502

Japan

Fax: +81-22-3087111

dq8m-kwmr@asahi-net.or.jp
License terms

(이요 $\Theta \circledast$
Background and study aims: Intramucosal vascular density differs between differentiated and undifferentiated type gastric carcinomas. This study aimed to evaluate the microvascular density characteristics of these two types of carcinoma using magnifying endoscopy with narrow-band imaging (ME-NBI).

Patients and methods: In total, 42 differentiated and 10 undifferentiated types were evaluated. The microvessels observed using ME-NBI were extracted from stored still images and the microvascular density in the two carcinoma types was analyzed. Histological vascular density in resected specimens was also evaluated using CD34 immunostaining.

Results: There were significant differences between the microvascular density in the differentiated and undifferentiated types of carcinoma $(10.02 \pm 4.72 \%$ vs $4.02 \pm 0.40 \% ; P<0.001)$ using ME-NBI. Vascular density assessed histologically also differed significantly between differentiated and undifferentiated types in both the whole mu-

\section{Introduction}

$\nabla$

In the Japanese classification, gastric carcinoma is generally divided into two pathological types, the differentiated type and the undifferentiated type, according to the presence of histological tubular structures [1]; these two pathological types correspond closely to the intestinal type and diffuse type of gastric carcinoma, respectively, in Lauren's classification [2]. Determination of carcinoma differentiation is considered to play a significant role in the treatment strategy; this is because, in the case of the undifferentiated type of gastric carcinoma, there is a higher possibility of lymph node metastasis than is the case for the differentiated type [3].

Several histopathological studies have suggested a correlation between carcinoma differentiation and intramucosal vascularity. The vascularity of cosal $(5.81 \pm 3.17 \%$ vs $3.25 \pm 1.21 \%)$ and the superficial mucosal layers $(0-100 \mu \mathrm{m})(6.38 \pm 3.73 \%$ vs $3.66 \pm 1.46 \%$ ). However, the vascular density in the surrounding non-carcinomatous mucosa assessed using ME-NBI and histologically, was significantly lower in the differentiated than in the undifferentiated types $(P<0.001)$. There was good agreement between ME-NBI and histologically assessed microvascular density in both the whole $(r=0.740 ; P<0.001)$ and superficial mucosal layers $(r=0.764 ; P<0.001)$. White opaque substance (WOS) was seen in eight patients who had the differentiated type carcinoma. In almost all cases with WOS, the appearance of the carcinoma was discolored.

Conclusions: There was a close relationship between ME-NBI assessed microvascular density and histologically assessed vascular density in the mucosal layer. Microvascular density differed significantly between the differentiated and undifferentiated types of carcinoma assessed using ME-NBI.

the differentiated type carcinoma is generally higher than that of the undifferentiated type [4]. This differentiation of vascularity has been reported to influence factors such as the color of carcinoma observed using conventional endoscopy [5].

Narrow-band imaging (NBI) allows endoscopic observation of minute microsurface structure or microvessels in the gastric mucosa. In recent years, several studies have reported the advantages of magnifying endoscopy with NBI (ME-NBI) for Helicobacter pylori induced gastritis: the identification of small gastric tumors; differentiation of gastric carcinoma from gastritis; and delineation of the horizontal extent of gastric carcinoma before endoscopic treatment [6-10]. Nakayoshi et al. [11] reported that the "network pattern" is often observed in the differentiated type of gastric carcinoma; however, the "corkscrew pattern" is often 
observed in the undifferentiated type of gastric carcinoma. Although the form of the microvessels has been investigated with regard to the pathological differentiation of gastric carcinoma, microvascular density using ME-NBI observation has not been investigated in detail.

In the present study, we investigated the differences in characteristics of the density of microvessels observed using ME-NBI between the differentiated and undifferentiated types of gastric carcinoma. We also evaluated the histological vascular density using resected specimens.

\section{Materials and methods}

$\nabla$

This observational study was performed in a single endoscopy unit at a city hospital (Sendai City Hospital, Sendai, Miyagi, Japan); the study was conducted in accordance with the provisions of the Helsinki Declaration. Between April 2010 and July 2011, 74 consecutive patients with intramucosal or submucosal gastric carcinoma who underwent endoscopic resection or a surgical operation were enrolled. Before carcinoma treatment, the microvessels were observed using ME-NBI and the images stored in a digital filing system. The density of the microvessels was evaluated using the stored still images, and the microvascular density in the two carcinoma types was investigated. A total of 22 patients were excluded from the study because of inappropriate NBI images as a result of bleeding, ulceration, or excessive mucosal congestion. Patients with severe anemia or portal hypertension were also excluded. Patients diagnosed as having a mixture of the differentiated and undifferentiated types of gastric carcinoma were also excluded to eliminate complications. Finally, 52 patients were analyzed (42 had differentiated type and 10 had undifferentiated type carcinoma) in the study. All participating patients were supplied with a detailed explanation of the study protocol and provided written informed consent.

\section{Endoscopic procedure}

The endoscopic procedure was performed using a CV-260 video system (OLYMPUS Co., Tokyo, Japan) and a magnifying endoscope (Model H260Z; OLYMPUS). To obtain a clear view using ME-NBI, a black rubber attachment (MB-162; OLYMPUS) was fitted to the tip of the videoendoscope to ensure an appropriate distance between the lens and the mucosal surface. A single experienced endoscopist (M.K.) performed the endoscopic procedure. Before treatment of the carcinoma, the videoendoscope was inserted into the stomach, and the gastric disease was visualized using standard and magnifying views. ME-NBI observations were undertaken approaching the maximum range, and the images (approximately 10-50 images) were saved in a digital filing system. If there were several types of microvessel in the carcinoma, we analyzed the area with major microvascular forms in the lesion. The uninvolved mucosa surrounding the carcinoma was also examined using ME-NBI.

\section{Microvessel assessment using ME-NBI}

From the stored still ME-NBI images, we extracted microvessels by computer image processing using image-processing software (ImageJ, version 1.50d; Rasband WS. National Institutes of Health, Bethesda, Maryland, USA; http://imagej.nih.gov/ij/, 1997-2012). We split the original NBI image in the RGB channel and used the blue color channel to visualize the microvessels with higher contrast. Using the image, we visually determined the threshold value for extracting the microvessels. After binarization, we extracted the vessel area and calculated the ratio of microvessels in the field. We also carried out this process in the surrounding non-carcinomatous area and calculated the density of the microvessels. The ratio of ME-NBI assessed vascularity in the carcinoma $(\mathrm{C})$ to that of the surrounding non-carcinomatous mucosa $(\mathrm{N})$ was also analyzed in each case.

\section{Histopathological assessment}

The resected carcinoma specimens were fixed in buffered formalin and embedded in paraffin. After hematoxylin and eosin staining, two expert pathologists (H.N. and R.S.) who were blinded to the ME-NBI findings, assessed the specimens with regard to the differentiation of gastric carcinoma. Gastric carcinomas were classified as the differentiated type (with the presence of tubular structures), and the undifferentiated type (with the absence of tubular structures) in accordance with the Japanese Classification of Gastric Carcinoma [1].

We determined the histological site in relation to the stored ME-NBI image and assessed the intramucosal vessels using CD34 immunostaining. Using the stained specimens, we captured macroscopic images and extracted the vascular field from the image using computerized and manual techniques. Finally, we calculated the area of vascularity in both the carcinoma and the surrounding non-carcinomatous areas in the same manner as that used in the ME-NBI analysis. Because several studies have reported that microvessels observed using ME-NBI are related to the shallow area of the intramucosal layer [10], we analyzed the histological vascular density not only in the whole mucosal layer but also in the superficial mucosal layer $(0-100 \mu \mathrm{m})$.

\section{Statistical analysis}

Quantitative results are presented as the mean \pm standard deviation. The clinical characteristics were evaluated using the Student's $t$ test, Fisher's exact test or the chi-squared test with the Yates continuity correction. The Student's unpaired $t$ test was used for the evaluation of microvascular density using ME-NBI and histologically assessed vascular density. Pearson's correlation coefficient $(r)$ was used to study the correlations between ME-NBI assessed microvascular density and histologically assessed vascular density. The Mann-Whitney $U$ test was used for $\mathrm{C} / \mathrm{N}$ ratio analysis with regard to ME-NBI assessed microvascular density. Statistical analyses were performed using SPSS Statistics software version 20.0 (SPSS Inc., Chicago, Illinois, United States), with $P$ values of $<0.05$ being considered to be statistically significant.

\section{Results \\ $\nabla$}

The characteristics of the patients are detailed in Table 1 . The color of the carcinomas appeared mainly reddish in the differentiated type and discolored in the undifferentiated type $(\bullet$ Fig. 1 and Fig. 2). The ME-NBI assessed microvascular density was $10.02 \pm 4.72 \%$ in the differentiated type and $4.02 \pm 0.40 \%$ in the undifferentiated type. There was a statistically significant difference in ME-NBI assessed microvascular density between the differentiated and undifferentiated types of carcinoma $(P<0.001$; - Table 2).

The histologically assessed vascular density of the differentiated type carcinoma was $5.81 \pm 3.17 \%$ in the whole mucosal layer and $6.38 \pm 3.73 \%$ in the superficial mucosal layer. The vascular densi- 


\begin{tabular}{|llll} 
& $\begin{array}{l}\text { Differentiated type } \\
\mathbf{n = 4 2}\end{array}$ & $\begin{array}{l}\text { Undifferentiated type } \\
\mathbf{n = 1 0}\end{array}$ & P value \\
\hline Age, mean \pm SD, years & $73.3 \pm 9.0$ & $60.3 \pm 10.4$ & $<0.001$ \\
\hline Sex, male/female, $\mathrm{n}(\%)$ & $35(83.3): 7(16.7)$ & $5(50.0): 5(50.0)$ & 0.039 \\
\hline Size of tumor mean \pm SD, mm & $18.5 \pm 9.8$ & $12.1 \pm 5.5$ & 0.053 \\
\hline Macroscopic type, 0-IIa/0-IIb/0-IIc, $\mathrm{n}(\%)$ & $12(28.6): 4(9.5): 26(61.9)$ & $0(0): 1(10.0): 9(90.0)$ & 0.149 \\
\hline Location, upper/middle/lower, $\mathrm{n}(\%)$ & $5(11.9): 17(40.5): 20(47.6)$ & $1(10.0): 8(80.0): 1(10.0)$ & 0.065 \\
\hline Depth of invasion, pT1 m/pT1sm, $\mathrm{n}(\%)$ & $34(81.0): 8(19.0)$ & $9(90.0): 1(10.0)$ & 0.830 \\
\hline WOS, positive/negative, $\mathrm{n}(\%)$ & $8(19.0): 34(81.0)$ & $0(0): 10(10.0)$ & 0.311 \\
\hline Color of tumor, reddish/discolored, $\mathrm{n}(\%)$ & $31(73.8): 11(26.2)$ & $1(10.0): 9(90.0)$ & $<0.001$ \\
\hline
\end{tabular}

Table 1 Characteristics of the patients enrolled in the study.

SD, standard deviation; 0-Ila, superficial elevated; 0-IIb, superficial flat; 0-Ilc, superficial depressed; pT1 m, tumor confined to the mucosa; PT1sm, tumor invading the layer of the submucosa; WOS, white opaque substance.
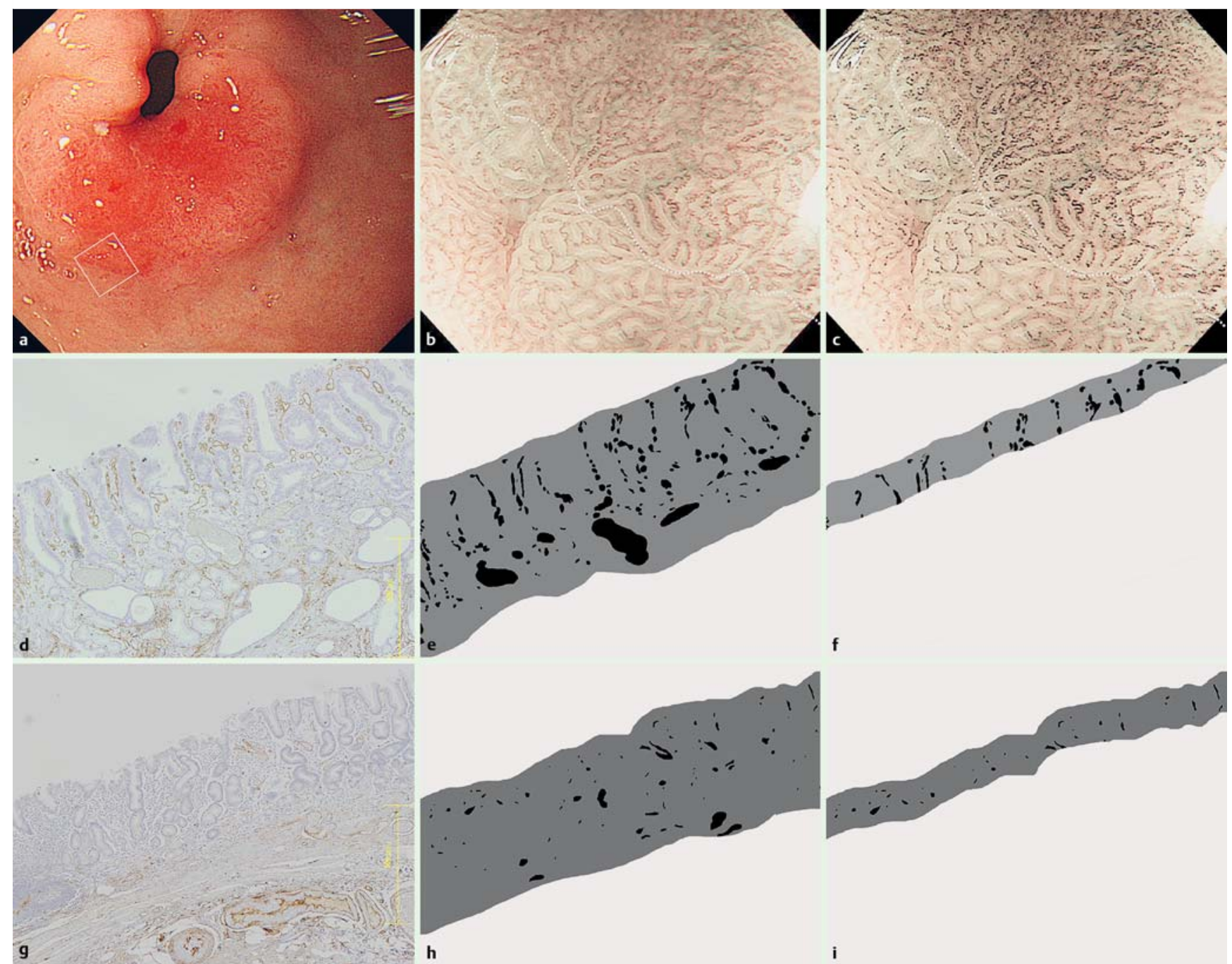

Fig. 1 a Reddish differentiated type gastric carcinoma in the antrum. b Magnifying endoscopy with narrow-band imaging (ME-NBI) (white box in a indicates location of $\mathbf{b}$; white dashed line: demarcation line of the carcinoma). $\mathbf{c}$ Microvessels extracted from the ME-NBI image. NBI vascular density was $11.23 \%$ in the carcinoma and $3.61 \%$ in the surrounding uninvolved mucosa (white dashed line: demarcation line of the carcinoma). $\mathbf{d}$ Photomicrograph showing a CD34 immunostained tumor tissue section. e Microvessels in the intramucosal layer were extracted. The histologically assessed vascular density for the carcinoma was $8.79 \%$ in the whole mucosal layer. $\mathbf{f}$ The histologically assessed vascular density was $9.26 \%$ in the superficial mucosal layer $(0-100 \mu \mathrm{m})$. $\mathbf{g}$ Photomicrograph showing a CD34 immunostained tissue section from the surrounding uninvolved mucosa. $\mathbf{h}$ The histologically assessed vascular density was $2.75 \%$ in the whole mucosal layer. I The histologically assessed vascular density was $2.18 \%$ in the superficial mucosal layer $(0-100 \mu \mathrm{m})$.

ties of the undifferentiated type carcinoma in the whole mucosal and superficial mucosal layers were $3.25 \pm 1.21 \%$ and $3.66 \pm 1.46 \%$, respectively. Both in the whole and superficial mucosal layers, the histologically assessed vascular density in the differentiated type was significantly higher than that in the undifferentiated type (whole mucosal layer, $P=0.016$; superficial mucosal layer, $P=$
0.029). However, with regard to the surrounding non-carcinomatous mucosa, both the ME-NBI and the histologically assessed vascular density of the non-carcinomatous mucosa surrounding the differentiated type carcinoma were significantly lower than the non-carcinomatous mucosa surrounding the undifferentiated type carcinoma $(P<0.001 ; 0$ Table 3$)$. There was a close relation- 

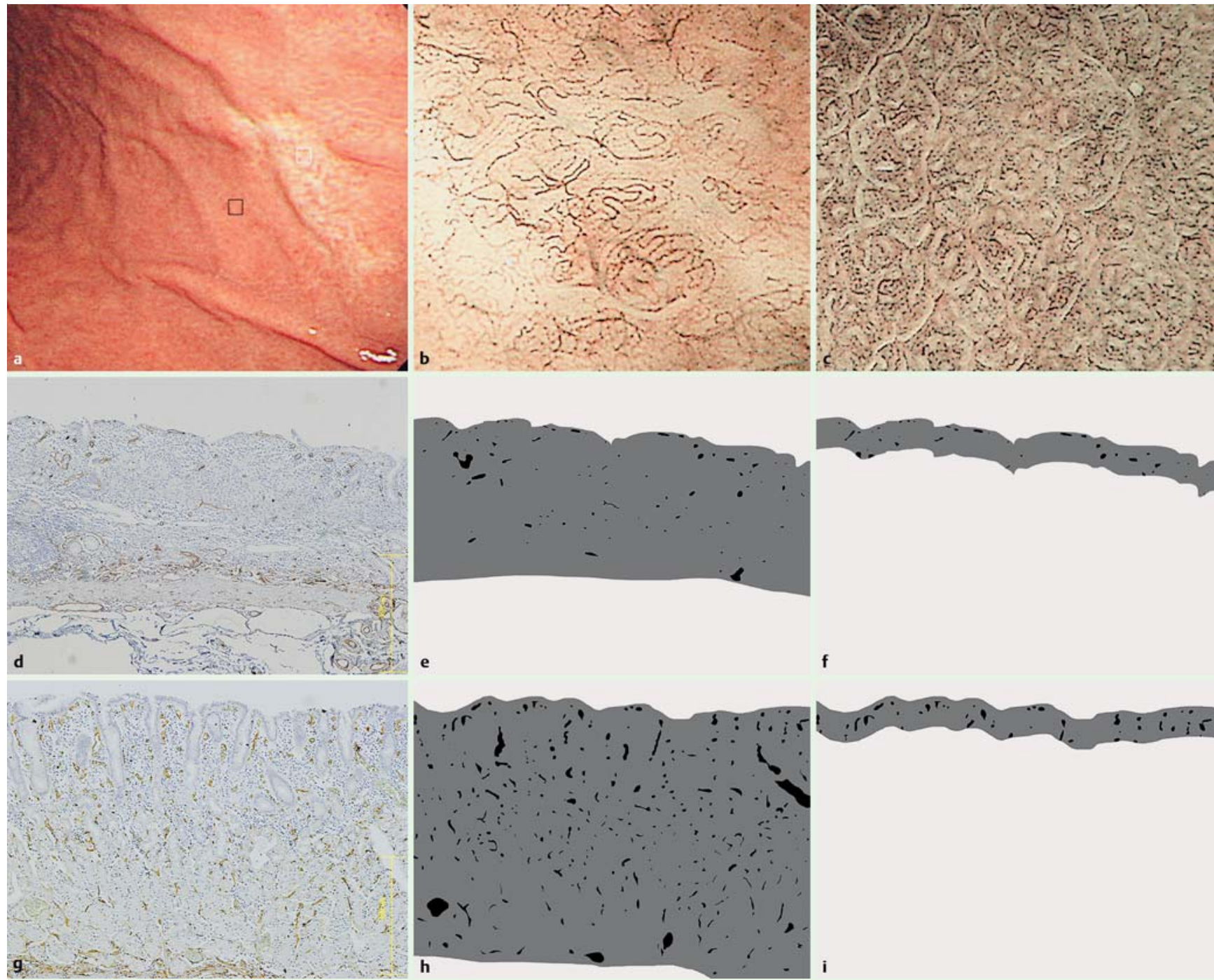

Fig. 2 a Discolored undifferentiated type gastric carcinoma in the middle corpus. b Microvessels were extracted from the ME-NBI image (white box in a indicates location of b). The NBI vascular density was $4.34 \%$ in the carcinoma. c The NBI vascular density was $10.59 \%$ in the surrounding uninvolved mucosa (black box in a indicates location of $\mathbf{c}$ ). $\mathbf{d}$ Photomicrograph showing a CD34 immunostained tissue section from a carcinoma. $\mathbf{e}$ The histologically assessed vascular index was $1.49 \%$ in the whole mucosal layer. $\mathbf{f}$ The histologically assessed vascular index was $2.56 \%$ in the superficial mucosal layer $(0-100 \mu \mathrm{m})$. g Photomicrograph showing a CD34 immunostained tissue section from the surrounding uninvolved mucosa. $\mathbf{h}$ The histologically assessed vascular density was $5.84 \%$ in the whole mucosal layer. I The histologically assessed vascular density was $6.87 \%$ in the superficial mucosal layer $(0-100 \mu \mathrm{m})$.

\begin{tabular}{|c|c|c|c|}
\hline & $\begin{array}{l}\text { Differentiated type } \\
\text { carcinoma }\end{array}$ & $\begin{array}{l}\text { Undifferentiated type } \\
\text { carcinoma }\end{array}$ & $P$ value \\
\hline NBI microvascular density, \% & $10.02 \pm 4.72$ & $4.02 \pm 0.40$ & $<0.001$ \\
\hline \multicolumn{4}{|l|}{ Histological vascular density, \% } \\
\hline Whole mucosal layer & $5.81 \pm 3.17$ & $3.25 \pm 1.21$ & 0.016 \\
\hline Surficial mucosal layer $(0-100 \mu \mathrm{m})$ & $6.38 \pm 3.73$ & $3.66 \pm 1.46$ & 0.029 \\
\hline
\end{tabular}

Table 2 Narrow-band imaging (NBI) assessment of microvascular density and histological assessment of vascular density in gastric carcinoma.

ship between ME-NBI assessed microvascular density and histologically assessed vascular density in both the whole and superficial mucosal layer (ME-NBI assessed whole mucosal layer, $r=0.740, P<0.001$; ME-NBI assessed superficial mucosal layer, $r=0.764, P<0.001)$. The $\mathrm{C} / \mathrm{N}$ ratio with regard to the ME-NBI assessed vascular density was significantly higher for the differentiated type carcinoma than for the undifferentiated type carcinoma $(P<0.001$; Table 4$)$. White opaque substance (WOS) was seen in eight patients who had the differentiated type carcinoma ( $\bullet$ Fig. 3); however, WOS was not seen in patients with the undifferentiated type carcinoma. In almost all cases with WOS (87.5\%), the appearance of the carcinoma was discolored.

\section{Discussion \\ $\nabla$}

To determine the treatment strategy for early gastric carcinoma, the histopathological differentiation of gastric carcinoma should be carefully considered. Endoscopic mucosal resection (EMR) is widely applied for minimally invasive treatment of early gastric carcinoma; however, undifferentiated type early gastric carcino- 


\begin{tabular}{|c|c|c|c|}
\hline & $\begin{array}{l}\text { Non-carcinomatous } \\
\text { mucosa surrounding } \\
\text { differentiated type } \\
\text { carcinoma }\end{array}$ & $\begin{array}{l}\text { Non-carcinomatous } \\
\text { mucosa surrounding } \\
\text { undifferentiated type } \\
\text { carcinoma }\end{array}$ & $P$ value \\
\hline NBI microvascular density, \% & $5.82 \pm 3.39$ & $10.99 \pm 3.94$ & $<0.001$ \\
\hline \multicolumn{4}{|l|}{ Histological vascular density, \% } \\
\hline Whole mucosal layer & $3.10 \pm 1.27$ & $7.40 \pm 2.51$ & $<0.001$ \\
\hline Surficial mucosal layer $(0-100 \mu \mathrm{m})$ & $2.49 \pm 1.11$ & $6.68 \pm 3.68$ & $<0.001$ \\
\hline
\end{tabular}

Table 3 Narrow-band imaging (NBI) assessment of microvascular density and histological assessment of vascular density in the surrounding non-carcinomatous mucosa.

\begin{tabular}{|llll}
\hline & $\begin{array}{l}\text { Differentiated type } \\
\text { carcinoma }\end{array}$ & $\begin{array}{l}\text { Undifferentiated type } \\
\text { carcinoma }\end{array}$ & P value \\
\hline C/N ratio of NBI vascular density & $3.94 \pm 8.22$ & $0.43 \pm 0.19$ & $<0.001$ \\
\hline
\end{tabular}

Table 4 C/N ratio for the microvascular density in the differentiated and undifferentiated types of carcinoma using NBI.

$\mathrm{C}$, carcinoma; $\mathrm{N}$, non-carcinomatous mucosa; $\mathrm{NBI}$, narrow-band imaging.

ma is not generally considered to be an indication for EMR, even using advanced endoscopic submucosal dissection techniques. The rate of lymph node metastasis has been demonstrated to be higher in intramucosal gastric carcinoma of the undifferentiated type ( $4.2 \%)$ than that in the differentiated type carcinoma $(0.4 \%)$ [3]. Thus, for those patients with a high risk factor concerning the development of lymph node metastasis, surgical gastrectomy with lymph node dissection should be indicated.

Regarding the determination of the pathological differentiation of gastric carcinoma, the vascularity of gastric carcinoma within the mucosa has been shown to differ in previous studies. Adachi et al. [4] examined the mucosal microvascular architecture of early gastric carcinoma using the Microfil injection method. They demonstrated that the diameter, length, and surface area of mucosal microvessels in differentiated type carcinoma was significantly higher than in undifferentiated type carcinoma. They also analyzed microvessels in the surrounding non-carcinomatous mucosa, and demonstrated that differentiated type carcinomas were mostly hypervascular or normovascular relative to the surrounding normal mucosa, while the undifferentiated type carcinomas were often hypovascular. This vascular differentiation in gastric carcinoma is considered to be related to the progression of tumor growth. The differentiated type carcinoma was accompanied by the proliferation of vessels within the neoplastic tissue, although the tumor cells in the undifferentiated type carcinoma exhibited sparse infiltration with destruction of the normal mucosal vascular architecture.

Clinically, the difference in mucosal vascularity influences the color change of gastric carcinoma viewed endoscopically [5]. Differentiated type gastric carcinomas often have a reddish color because of increased mucosal vascularity, while undifferentiated type gastric carcinomas often appear discolored because of decreased mucosal vascularity. The present study is the first to reveal that there was a difference between the microvascular density in the differentiated and undifferentiated types of gastric carcinoma assessed using ME-NBI. The results agree with those of previous histopathological vascularity analyses. Our findings have greater clinical relevance concerning the direct evaluation of microvessels compared with other studies such as those involving the $\mathrm{Hb}$ index calculation [12].

Using spectrally narrow band filters for selecting wavelengths that were absorbed well by hemoglobin, ME-NBI provides good visualization of microvessels in the superficial gastric mucosa $[13,14]$. In a previous report [11], the forms of microvessels observed using ME-NBI were useful markers with regard to the diagnosis of carcinoma differentiation. Our results revealed that not only the form but also the microvessel densities were useful markers. Our results indicate that it is necessary to more pay attention to lower density areas of ME-NBI detected microvessels.

In the present study, we investigated the whole mucosal vascular density histopathologically using CD34 immunostaining, and demonstrated good agreement with ME-NBI assessed microvascular density. Several histopathological studies involving mucosal vascularity in gastric carcinoma have evaluated intramucosal vascular density, using the average microvascular diameter of selected hot spot areas in the microscopic field. This calculation method is considered insufficient for the evaluation of whole mucosal blood flow, because vascularity consists of not only vessel diameter but also length or number of vessels. Selection of a hot spot of intramucosal vessels in the specimens is also inade-

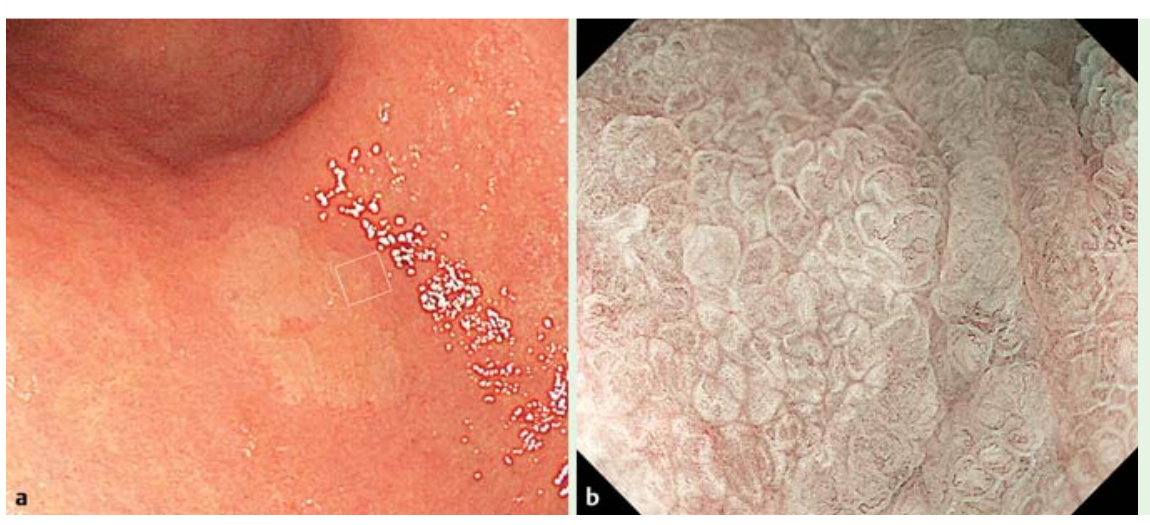

Fig. 3 a A discolored differentiated type gastric carcinoma in the antrum. $\mathbf{b}$ ME-NBI image showing white opaque substance in the carcinoma, obstructing the visualization of the microvessels (white box in a indicates location of $\mathbf{b}$ ). 
quate for evaluating mucosal vascularity because of limited field size. We believe that our method is sufficiently robust in evaluating the relationship between endoscopically assessed microvascular density and histologically assessed vascularity; however, we should consider the fact that these histopathological images (vertical view) were not equivalent to the endoscopic images (horizontal view) as a limitation of the study.

In recent studies involving ME-NBI, WOS was sometimes seen in the superficial area of gastric neoplasia $[15,16]$. WOS that is related to the accumulation of tiny lipid droplets, obstructs the visualization of the microvessels. In our study, WOS was seen in eight patients who all had the differentiated type carcinoma; however, WOS was not seen in patients with the undifferentiated type carcinoma. In almost all cases with WOS, the appearance of the carcinoma was discolored; thus, WOS is an important factor influencing the color of the carcinoma. Our methods were inadequate for the investigation of carcinomas with WOS, because it was impossible to make a diagnosis from the microvascular density or microvascular using ME-NBI.

A limitation of our study was the small number of patients enrolled, especially those with undifferentiated type carcinomas. The lack of analysis of mixed histological types was also an additional limitation. We revealed a significant difference in the ME-NBI assessed vascular density between differentiated and undifferentiated types of gastric carcinoma; however, further study is required to determine whether they have value in the selection of treatment strategies, especially in comparison with the "goldstandard" biopsy diagnosis. The present study was not blinded; thus, ME-NBI observation was mainly performed after the diagnosis of histological type using the biopsy procedure. A prospective multicenter study involving the appropriate number of ME-NBI images will be required to confirm whether our results are applicable to daily clinical practice.

In conclusion, we demonstrated a good correlation between ME-NBI and pathologically assessed vascular density in the superficial and whole mucosal layers. We also revealed a significant difference in the ME-NBI assessed vascular density between differentiated and undifferentiated types of gastric carcinoma.

Competing interests: None

\section{References}

1 Japanese Gastric Cancer Association. Japanese classification of gastric carcinoma: 3rd English edition. Gastric Cancer 2011; 14: 101 - 112

2 Lauren $P$. The two histological main types of gastric carcinoma: Diffuse and so-called intestinal-type carcinoma. An attempt at a histo-clinical classification. Acta Pathol Microbiol Scand 1965; 64: 31 - 49

3 Gotoda $T$, Yanagisawa A, Sasako $M$ et al. Incidence of lymph node metastasis from early gastric cancer: estimation with a large number of cases at two large centers. Gastric Cancer 2000; 3: 219-225

4 Adachi $Y$, Mori $M$, Enjoji $M$ et al. Microvascular architecture of early gastric carcinoma. Microvascular-histopathologic correlates. Cancer 1993; 72: 32-36

5 Honmyo U, Misumi A, Murakami A et al. Mechanisms producing color change in flat early gastric cancers. Endoscopy 1997; 29: 366-371

6 Yagi K, Nakamura A, Sekine A. Comparison between magnifying endoscopy and histological, culture and urease test findings from the gastric mucosa of the corpus. Endoscopy 2002; 34: 376-381

7 Kawamura M, Abe S, Oikawa K et al. Topographic differences in gastric micromucosal patterns observed by magnifying endoscopy with narrow band imaging. J Gastroenterol Hepatol 2011; 26: 477-483

8 Ezoe $Y$, Muto $M$, Uedo $N$ et al. Magnifying narrowband imaging is more accurate than conventional white-light imaging in diagnosis of gastric mucosal cancer. Gastroenterology 2011; 141: 2017-2025

9 Nagahama T, Yao K, Maki S et al. Usefulness of magnifying endoscopy with narrow-band imaging for determining the horizontal extent of early gastric cancer when there is an unclear margin by chromoendoscopy (with video). Gastrointest Endosc 2011; 74: 1259-1267

10 Pimentel-Nunes P, Dinis-Ribeiro M, Soares JB et al. A multicenter validation of an endoscopic classification with narrow band imaging for gastric precancerous and cancerous lesions. Endoscopy 2012; 44: 236 246

11 Nakayoshi T, Tajiri H, Matsuda K et al. Magnifying endoscopy combined with narrow band imaging system for early gastric cancer. Endoscopy 2004; 36: 1080 - 1084

12 Yao K, Yao T, Matsui T et al. Hemoglobin content in intramucosal gastric carcinoma as a marker of histologic differentiation: a clinical application of quantitative electronic endoscopy. Gastrointest Endosc 2000; 52: $241-245$

13 Gono K, Obi T, Yamaguchi $M$ et al. Appearance of enhanced tissue features in narrow-band endoscopic imaging. J Biomed Opt 2004; 9: $568-577$

14 Yagi $K$, Nozawa $Y$, Endou $S$ et al. Diagnosis of early gastric cancer by magnifying endoscopy with NBI from viewpoint of histological imaging: mucosal patterning in terms of white zone visibility and its relationship to histology. Diagn Ther Endosc 2012; 2012: 954809

15 Yao K, Iwashita A, Tanabe $H$ et al. White opaque substance within superficial elevated gastric neoplasm as visualized by magnification endoscopy with narrow-band imaging: a new optical sign for differentiating between adenoma and carcinoma. Gastrointest Endosc 2008; 68: $574-580$

16 Yao K, Iwashita A, Nambu M et al. Nature of white opaque substance in gastric epithelial neoplasia as visualized by magnifying endoscopy with narrow-band imaging. Dig Endosc 2012; 24: 419-425 